ARTICLE

https://doi.org/10.1038/s41467-019-10435-2

\title{
Impact of molecular quadrupole moments on the energy levels at organic heterojunctions
}

\author{
Martin Schwarze (1) ${ }^{1}$, Karl Sebastian Schellhammer ${ }^{2,3}$, Katrin Ortstein (1) 1, Johannes Benduhn (1) 1, \\ Christopher Gaul (1) ${ }^{3}$, Alexander Hinderhofer ${ }^{4}$, Lorena Perdigón Toro (1) ${ }^{5}$, Reinhard Scholz ${ }^{1}$, Jonas Kublitski (1) ${ }^{1}$, \\ Steffen Roland ${ }^{5}$, Matthias Lau', Carl Poelking', Denis Andrienko (iD) ${ }^{6}$, Gianaurelio Cuniberti (i) ${ }^{2}$, Frank Schreiber ${ }^{4}$, \\ Dieter Neher (1) ${ }^{5}$, Koen Vandewal (10) ${ }^{1,7}$, Frank Ortmann (i) ${ }^{3}$ \& Karl Leo (i) ${ }^{1}$
}

The functionality of organic semiconductor devices crucially depends on molecular energies, namely the ionisation energy and the electron affinity. Ionisation energy and electron affinity values of thin films are, however, sensitive to film morphology and composition, making their prediction challenging. In a combined experimental and simulation study on zincphthalocyanine and its fluorinated derivatives, we show that changes in ionisation energy as a function of molecular orientation in neat films or mixing ratio in blends are proportional to the molecular quadrupole component along the $\pi$ - $\pi$-stacking direction. We apply these findings to organic solar cells and demonstrate how the electrostatic interactions can be tuned to optimise the energy of the charge-transfer state at the donor-acceptor interface and the dissociation barrier for free charge carrier generation. The confirmation of the correlation between interfacial energies and quadrupole moments for other materials indicates its relevance for small molecules and polymers.

\footnotetext{
${ }^{1}$ Dresden Integrated Center for Applied Physics and Photonic Materials (IAPP), Technische Universität Dresden, 01069 Dresden, Germany. ${ }^{2}$ Institute for Materials Science, Max-Bergmann Center of Biomaterials and Dresden Center for Computational Materials Science, Technische Universität Dresden, 01069 Dresden, Germany. ${ }^{3}$ Center for Advancing Electronics Dresden, Technische Universität Dresden, 01069 Dresden, Germany. ${ }^{4}$ Institute of Applied Physics, University of Tübingen, Auf der Morgenstelle 10, 72076 Tübingen, Germany. ${ }^{5}$ Institute of Physics and Astronomy, University of Potsdam, Karl-LiebknechtStr. 24-25, 14476 Potsdam, Germany. ${ }^{6}$ Max Planck Institute for Polymer Research, Ackermannweg 10, 55128 Mainz, Germany. ${ }^{7}$ Present address: Instituut voor Materiaalonderzoek (IMO), Hasselt University, Wetenschapspark 1, 3590 Diepenbeek, Belgium. Correspondence and requests for materials should be addressed to M.S. (email: Martin.Schwarze1@tu-dresden.de) or to F.O. (email: Frank.Ortmann@tu-dresden.de) or to K.L. (email: karl.leo@iapp.de)
} 
O rganic semiconductors gained much attention because of their attractive application in low-cost, large area, and flexible electronic devices ${ }^{1-4}$. While organic lightemitting diodes (OLEDs) already entered the market in thin film displays, several other promising applications such as solar cells, transistors, photodetectors, or lasers still require improvements in performance. In contrast to their inorganic counterparts, organic semiconductors typically consist of weakly bound molecules, where charge carriers occupy rather localised states. Associated to these states are the ionisation energy (IE) and electron affinity (EA) of organic molecules, which are related to the transport energies of holes and electrons and, consequently, determine the functionality of electronic devices ${ }^{5,6}$.

In organic solar cells (OSCs), fundamental processes determining the device performance are the dissociation of chargetransfer (CT) states at the donor-acceptor interface into free charges and their non-geminate recombination via $\mathrm{CT}$ states back to the ground state ${ }^{7-9}$. Therefore, the CT state energy $\left(E_{\mathrm{CT}}\right)$ determines the open-circuit voltage $\left(V_{\text {oc }}\right)$ of OSCs ${ }^{10}$, while its difference to the energy of separated charges $\left(E_{\mathrm{CS}}\right)$ influences the generation efficiency of free charge carriers and, thus, crucially affects both the short-circuit current density $\left(j_{\mathrm{sc}}\right)$ and the fillfactor $(\mathrm{FF})^{11,12}$. Both $E_{\mathrm{CT}}$ and $E_{\mathrm{CS}}$ are linked to IE of the donor and EA of the acceptor.

IE and EA of molecules in organic films significantly depend on molecular orientation and mixing ratio in blends ${ }^{13-15}$. In particular, charge-quadrupole interaction can induce large electrostatic shifts of the electronic levels in crystalline films ${ }^{16-19}$, which, for example, allows in blends with molecular intermixing, a continuous tuning of IE and EA by adjusting the ratio of two different molecular species ${ }^{20,21}$. Furthermore, simulations indicated that these interactions can assist the dissociation of CT states at planar donor-acceptor interfaces ${ }^{22,23}$. However, it remains an open question to which extent such findings for these model systems are general and, particularly, how they are applicable to donor:acceptor blends without long-range order which are usually employed in efficient OSCs.

In this study, we demonstrate the tunability of the solid-state IE by charge-quadrupole interactions and their relevance in systems with long-range and short-range order, being, thus, relevant for most organic devices. As a model system, we choose zincphthalocyanine $(\mathrm{ZnPc})$ because of the possibility to gradually change its quadrupole moment (QPM) by stepwise fluorination $\left(\mathrm{F}_{n} \mathrm{ZnPc}\right)^{20}$. In order to establish the role of the quadrupole component perpendicular to the molecular plane $\left(Q_{\pi}\right)$, we measure for $\mathrm{F}_{n} \mathrm{ZnPc}$ the change in IE with molecular orientation, film thickness, and mixing ratio in blends. The ultraviolet photoelectron spectroscopy (UPS) analysis reveals a linear change of IE with $Q_{\pi}$ in all cases. Moreover, when applying these findings to OSCs, we demonstrate how QPMs influence $E_{\mathrm{CT}}$ at planar and bulk heterojunctions between donor and acceptor. Time-delayed collection field (TDCF) measurements further show that electrostatic gradients induced by QPMs can assist free charge carrier generation in these solar cells. Finally, we extend the study to other material systems, indicating the relevance of the findings for a large variety of organic semiconductors.

\section{Results}

Dependence of thin flm energies on the component $Q_{\pi}$. IE and EA of molecules in thin films deviate from their gas-phase values, $\mathrm{IE}_{0}$ and $\mathrm{EA}_{0}$, due to polarisation effects ${ }^{24-26}$. In weakly bound solids with localised states, the electrostatic corrections $\left(\Delta_{+}\right.$and $\left.\Delta_{-}\right)$to the gas-phase values consist of an induced and a permanent contribution. While the first term decreases the distance between IE and EA, the latter originates from the interaction of excess charges with static charge distributions and shifts IE and EA equally ${ }^{17-20}$. We concentrate on the permanent contribution in this study, which is often dominated by the charge-quadrupole term in the multipole expansion because molecules with dipole moments often stack with alternating molecular orientations in ordered organic solids ${ }^{16,18}$. In this case, the permanent contribution can be approximated by a sum over the interaction energies of a charged molecule, described by its atomic excess charges $q_{j}$ at positions $\mathbf{r}_{j}$, with the quadrupole tensors $\mathbf{Q}_{i}$ of all surrounding molecules at sites $\mathbf{r}_{i}^{27}$

$$
E_{\mathrm{Q}}=\sum_{i, j} \frac{q_{j}}{8 \pi \epsilon_{0} \epsilon_{\mathrm{r}}} \cdot \frac{\left(\mathbf{r}_{i}-\mathbf{r}_{j}\right) \cdot \mathbf{Q}_{i} \cdot\left(\mathbf{r}_{i}-\mathbf{r}_{j}\right)}{\left|\mathbf{r}_{i}-\mathbf{r}_{j}\right|^{5}},
$$

where we use the dielectric permittivity $\varepsilon_{\mathrm{r}}$ as a macroscopic constant. We describe the charged molecule by its actual distribution of atomic charges to have an appropriate description at distances in the range or below the spatial extent of molecules.

Due to the strong dependence of $E_{\mathrm{Q}}$ on distance, $E_{\mathrm{Q}}$ changes with the chemical and crystal structure of the compound. Like many other planar molecules, $\mathrm{F}_{n} \mathrm{ZnPc}$ typically arranges in a $\pi$ $-\pi$-stacking geometry 28,29 , where the intermolecular distance along the stacking direction (approximately $3.8 \AA^{28}$ ) is significantly smaller than along the other two directions $\left(13-14.5 \AA^{30}\right)$. Therefore, we first investigate if the quadrupole component perpendicular to the molecular plane $\left(Q_{\pi}\right)$ dominates $E_{\mathrm{Q}}$ (see Fig. 1 for $Q_{\pi}$ values) by analysing for $\mathrm{F}_{n} \mathrm{ZnPc}$ layers $(20 \mathrm{~nm})$ the difference in IE between face-on and edge-on orientation of the molecules (Fig. 2a, see Supplementary Figs. 1-3 and Supplementary Note 1 for X-ray scattering results). The representative UPS spectra in Fig. $2 \mathrm{~b}$ show that IE of a $\mathrm{ZnPc}$ film in edge-on orientation is $0.22 \mathrm{eV}$ smaller as compared to the film in face-on orientation, in agreement with previous results ${ }^{13}$. In contrast, $\mathrm{F}_{8} \mathrm{ZnPc}$ exhibits an IE value in edge-on orientation that is $0.37 \mathrm{eV}$ larger than the IE value in face-on orientation. To connect this behaviour to molecular properties, we calculate $Q_{\pi}$ by density functional theory (DFT) for $\mathrm{ZnPc}$ and its fluorinated derivatives (results in Supplementary Table 1). As displayed in Fig. 2c, the difference in IE between edge-on and face-on orientation increases with $Q_{\pi}$ from $\mathrm{ZnPc}$ to $\mathrm{F}_{16} \mathrm{ZnPc}$.

To prove that $Q_{\pi}$ dominates in $E_{\mathrm{Q}}$ (see Eq. 1), we calculate $E_{\mathrm{Q}}$ for a single charged molecule at the film surface for both orientations (see Methods and Supplementary Fig. 4). The difference in $E_{\mathrm{Q}}$ between both orientations exhibits the same correlation with $Q_{\pi}$ as observed for the IE difference in experiment (Fig. 2c). Interestingly, the simulations reveal that the relevant range of charge-quadrupole interactions is different between both molecular orientations (Supplementary Fig. 5). For edge-on orientation, $E_{\mathrm{Q}}$ is dominated by interactions between the next few neighbours along the $\pi-\pi$-stacking geometry. For faceon orientation, $E_{\mathrm{Q}}$ is also dominated by interactions with $Q_{\pi}$ components at small integration limits, which however is compensated by the interaction with other components when the integration limit in the lateral direction becomes larger than $100 \mathrm{~nm}$. Previous investigations indicate that the interaction with other components than $Q_{\pi}$ can be even more dominant in face-on orientation for molecules with a different symmetry than $\mathrm{F}_{n} \mathrm{ZnPc}$ such as pentacene, having two molecular short axes ${ }^{19,31}$. In the absence of long-range order, such as in donor:acceptor blends with crystal sizes much smaller than $100 \mathrm{~nm}$, the interaction of charges with $Q_{\pi}$ components should dominate ${ }^{32-34}$.

To further verify the dominance of charge-quadrupole interactions along the $\pi-\pi$-stacking direction for edge-on orientation, we measure IE of $\mathrm{F}_{n} \mathrm{ZnPc}$ for coverages below the monolayer thickness and evaporate the material stepwise onto 

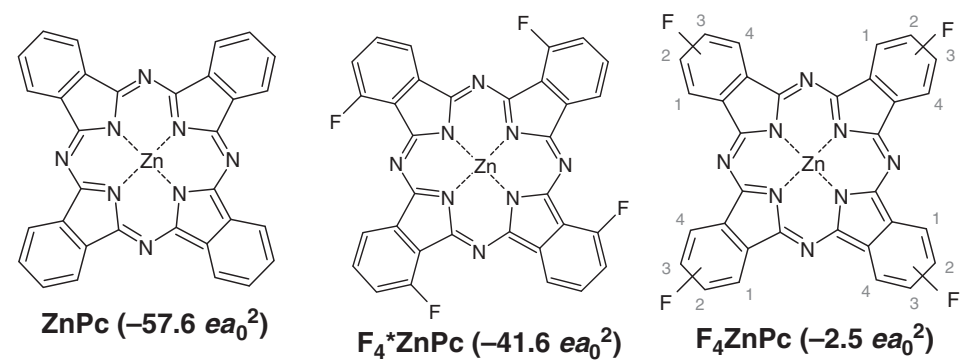

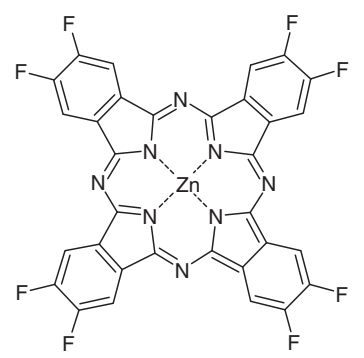

$\mathrm{F}_{8} \mathrm{ZnPc}\left(49.0{\left.e a_{0}{ }^{2}\right)}^{2}\right.$

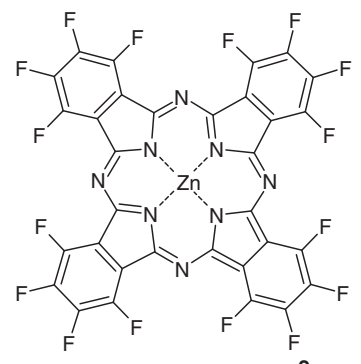

$F_{16} \mathrm{ZnPc}\left(72.0 \mathrm{ea}_{0}{ }^{2}\right)$

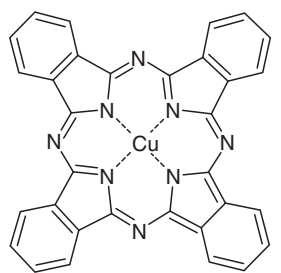

CuPc (-59.8 ea $\left.{ }^{2}\right)$

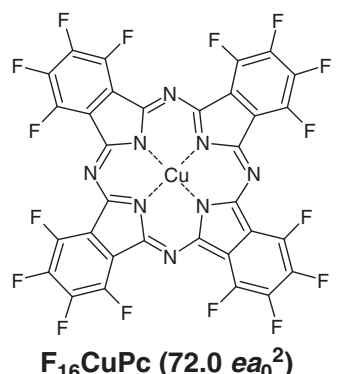<smiles>Fc1cc2nc3c4nc5cc(F)c(F)cc5nc4c4nc5cc(F)c(F)cc5nc4c3nc2cc1F</smiles>
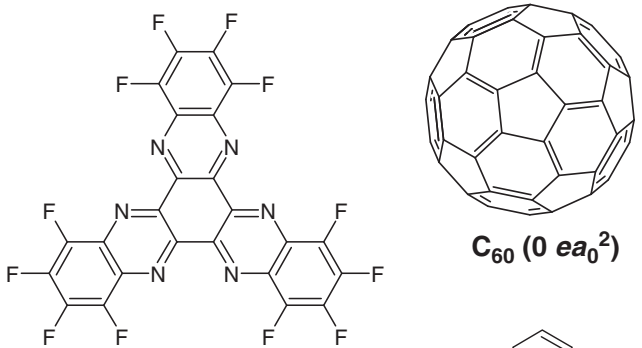

$\mathrm{C}_{60}\left(0 \mathrm{e} \mathrm{a}_{0}{ }^{2}\right)$

HATNA-F $6\left(21.8 e a_{0}^{2}\right)$
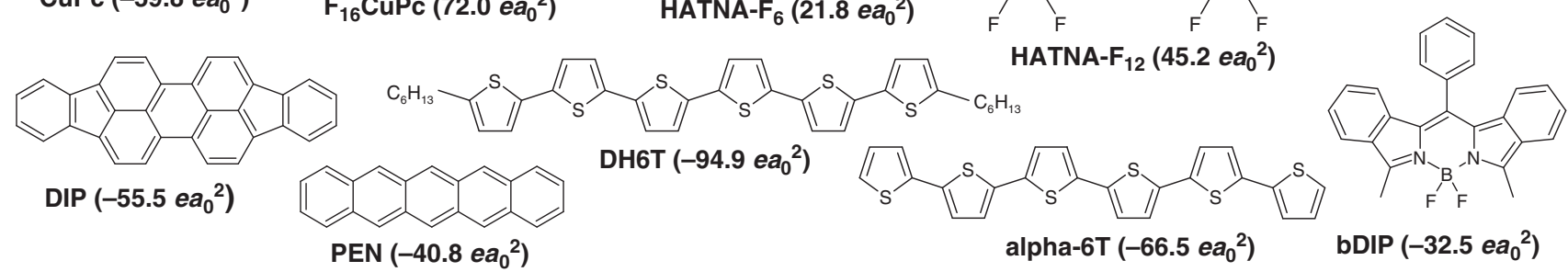

alpha-6T (-66.5 $\left.e a_{0}^{2}\right)$

bDIP $\left(-32.5 e a_{0}^{2}\right)$

Fig. 1 Chemical structures and quadrupole components of small molecules: Values in brackets are the quadrupole components perpendicular to the molecular plane $\left(Q_{\pi}\right)$, as calculated with density functional theory (DFT). Supplementary Table 1 summarises the in-plane quadrupole components. $F_{4} Z n P c$ is a mixture of different isomers, where the fluorine atom is bonded to one of the two outer carbon positions ( 2 or 3$)^{20}$

p-doped BPAPF, leading to an edge-on orientation in thin films (Supplementary Figure 6). IE at very small coverages, denoted as $\mathrm{IE}_{\mathrm{s}}$, deviates strongly from the value of thick layers (see dotted lines in Fig. 2d). Notably, for all $\mathrm{ZnPc}$ derivatives, $\mathrm{IE}_{\mathrm{s}}$ is reduced by $0.6 \mathrm{eV}$ compared to their respective gas-phase ionisation energies $\mathrm{IE}_{0}$, indicating minor influence of charge-quadrupole interactions on IE at low coverages. With increasing layer thickness, IE strongly changes and approaches the value of a thick edge-on oriented film when the monolayer thickness $\left(13-14.5 \AA^{30}\right)$ is reached. The strong IE shift can be explained by the formation of an ordered monolayer in edge-on orientation, leading to an increase of the magnitude of $E_{\mathrm{Q}}$ due to charge-quadrupole interactions along the $\pi-\pi$-stacking direction. In good agreement, the change of IE scales with the $Q_{\pi}$ value of the respective $\mathrm{ZnPc}$ derivative (Supplementary Fig. $6 \mathrm{c}$ ). Note that the change in IE until the first edge-on monolayer is formed can occur for different growth modes, such as island growth or transition of molecular orientation from face-on to edge-on.

After having demonstrated the strong effect of $Q_{\pi}$ on the energy levels in model systems based on $\mathrm{ZnPc}$ derivatives, we further analyse its impact in donor:acceptor blends which are typically used in OSCs. Such blended structures usually exhibit significant structural disorder and phase separation that both can change with mixing ratio ${ }^{13,32,35,36}$. Previous studies found changes of energy levels with donor:acceptor mixing ratio, which were assigned to changes in crystal size and to electrostatic changes $13,15,37$. We trace back these energetic changes to the influence of the molecular tuning parameter $Q_{\pi}$.

We measure the energy levels of $\mathrm{F}_{8} \mathrm{ZnPc}: \mathrm{C}_{60}$ blends at different mixing ratios (see UPS spectra in Supplementary Fig. 7). As shown in Fig. 3a, IE of $\mathrm{F}_{8} \mathrm{ZnPc}$ decreases by more than $300 \mathrm{meV}$ with increasing $\mathrm{C}_{60}$ content. IE of $\mathrm{C}_{60}$ shows a similar change, which suggests that a large amount of donor and acceptor molecules interact electrostatically with the other species despite the phase separation in these blends. The larger IE change of $\mathrm{F}_{8} \mathrm{ZnPc}$ at higher $\mathrm{C}_{60}$ contents indicates that $\mathrm{F}_{8} \mathrm{ZnPc}$ molecules which are closer to $\mathrm{C}_{60}$ molecules experience a larger electrostatic shift.

To validate that the electrostatic changes originate from charge -quadrupole interactions, we compare the behaviour of $\mathrm{F}_{8} \mathrm{ZnPc}$ : $\mathrm{C}_{60}$ blends to previously reported $\mathrm{ZnPc}: \mathrm{C}_{60}$ blends ${ }^{13}$, as $\mathrm{ZnPc}$ and $\mathrm{F}_{8} \mathrm{ZnPc}$ exhibit a similar magnitude but a different sign of $Q_{\pi}$. Figure $3 \mathrm{~b}$ shows the electrostatic correction $\Delta_{+}$of IE for $\mathrm{ZnPc}$ and $\mathrm{F}_{8} \mathrm{ZnPc}$ blended with $\mathrm{C}_{60}$. While $\Delta_{+}$differs strongly between $\mathrm{ZnPc}$ and $\mathrm{F}_{8} \mathrm{ZnPc}$ in pure layers, this difference reduces continuously with increasing $\mathrm{C}_{60}$ concentration, and $\Delta_{+}$finally approaches $-0.6 \mathrm{eV}$. This value is also observed for very small coverages of $\left(\mathrm{F}_{8}\right) \mathrm{ZnPc}$, indicating that the charge-quadrupole interaction energy $E_{\mathrm{Q}}$ approaches 0 for high $\mathrm{C}_{60}$ contents (see Fig. 2d for comparison). Therefore, we attribute the decrease (increase) of IE of $\mathrm{F}_{8} \mathrm{ZnPc}(\mathrm{ZnPc})$ with increasing $\mathrm{C}_{60}$ content to the interaction of charges with a reduced number of $Q_{\pi}$ components of the donor.

The dominance of charge-quadrupole interactions along the $\pi$ $-\pi$-stacking direction is attributed to the shorter intermolecular distance in this direction for the $\mathrm{ZnPc}$ derivatives. To verify whether this simple model is also valid for other materials, we extend our investigation to other molecules that show a strong dependence of IE on molecular orientation in neat films ${ }^{13,14,38-41}$ or on mixing ratio in donor:acceptor blends ${ }^{15,37}$ (see Fig. 1 for chemical structures). We calculate their QPMs and observe that for both cases the change in IE scales linearly with the respective $Q_{\pi}$ (Supplementary Fig. 8). Notably, the slope is surprisingly similar to the one observed for intermixed blends of different ZnPc derivatives ${ }^{20}$ (Supplementary Fig. 9). Charge-quadrupole interactions are also relevant for polymers. Similar to 
a
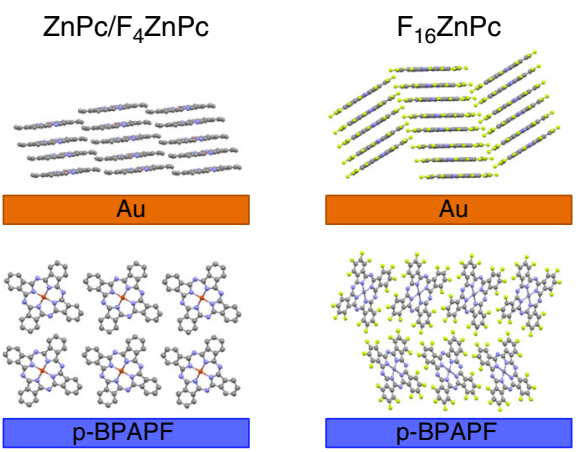

b

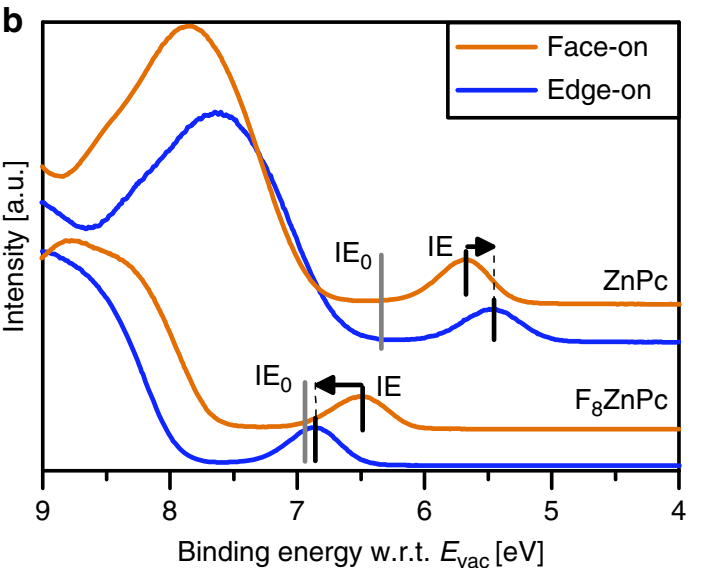

C
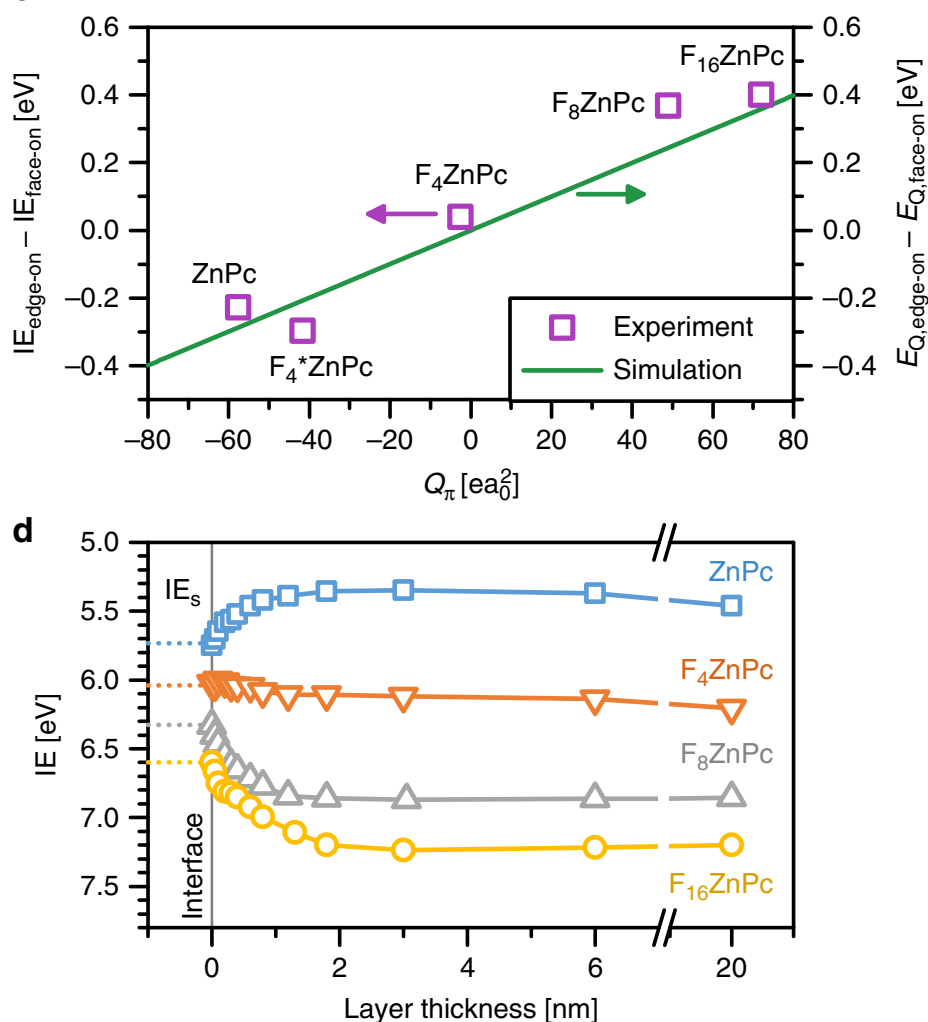

Fig. 2 Dependence of IE on charge-quadrupole interactions along the $\pi-\pi$-stacking geometry: a Sketch of the film structure of $\mathrm{ZnPc}, \mathrm{F}_{4} \mathrm{ZnPc}$ and $\mathrm{F}_{16} \mathrm{ZnPc}$, showing the difference in molecular orientation between two different substrates. $\mathbf{b}$ UPS spectra of thin films of $\mathrm{ZnPc}\left(\right.$ top) and $\mathrm{F}_{8} \mathrm{ZnPc}($ bottom) in edgeon orientation (substrate: p-doped BPAPF on silver) and face-on orientation (substrate: gold). The different quadrupole moments of $\mathrm{ZnPc}$ and $\mathrm{F}_{8} \mathrm{ZnPc}$ cause electrostatic shifts in opposite directions from the gas-phase IE (IE

c The difference in IE between edge-on and face-on orientation in UPS experiments (violet squares) scales with the calculated quadrupole component perpendicular to the molecular plane $\left(Q_{\pi}\right)$. The green solid line shows the difference in the charge-quadrupole interaction energy $E_{Q}$ between both orientations, as obtained from simulation. $\mathbf{d}$ IE of $\mathrm{F}_{n} \mathrm{ZnPc}$ on $\mathrm{p}$-doped BPAPF, obtained by subtracting the substrate spectrum from the superimposed spectra (see Supplementary Fig. 6a), changes strongly during the formation of the first monolayer in edge-on orientation due to charge-quadrupole interactions along the $\pi-\pi$-stacking direction. The slight changes of IE from 3 to $20 \mathrm{~nm}$ are also observed in simulation and can be explained by increased interactions with other quadrupole components than $Q_{\pi} \cdot \mathrm{IE}_{\mathrm{s}}$ (dotted lines) is attributed to molecules with $E_{\mathrm{Q}} \approx 0$, being $0.6 \mathrm{eV}$ smaller than $\mathrm{IE}_{0}$

sexithiophene (alpha-6T), poly-3-hexylthiophene (P3HT) shows a smaller IE for end-on orientation with the polymer chain perpendicular to the substrate plane ${ }^{42}$. This analysis indicates that the impact of $Q_{\pi}$ on thin film energy levels is relevant for many organic materials including varying molecular structures and different morphologies of their (blend) films.

Impact of $Q_{\pi}$ at donor-acceptor interfaces in solar cells. The functionality of OSCs is linked to the formation of chargetransfer (CT) states of a donor cation and an acceptor anion at their interface (Fig. 4a), mediating charge carrier dissociation and recombination in OSCs ${ }^{7,8}$. In the following, we demonstrate how molecular quadrupole moments can influence the CT state energy $\left(E_{\mathrm{CT}}\right)$ as well as its difference to the energy of separated charges $\left(E_{\mathrm{CS}}\right)$. For this purpose, we analyse $\mathrm{ZnPc}: \mathrm{F}_{4} \mathrm{ZnPc}: \mathrm{C}_{60}$ solar cells based on bulk heterojunctions $(\mathrm{BHJ})$ with two electron donating molecules ( $\mathrm{ZnPc}$ and $\mathrm{F}_{4} \mathrm{ZnPc}$ ) and one acceptor $\left(\mathrm{C}_{60}\right)$ with a fixed volume content of the acceptor $(60 \%)$. Different mixing ratios between $\mathrm{ZnPc}$ and $\mathrm{F}_{4} \mathrm{ZnPc}$ are used to selectively change the average of the molecular parameters in the donor phase.

For analysing $E_{\mathrm{CT}}$, we obtain the relevant gas-phase energy levels of donor and acceptor molecules $\left(\mathrm{IE}_{0, \mathrm{D}}\right.$ and $\mathrm{EA}_{0, \mathrm{~A}}$ ) as well as the intramolecular relaxation energies of their ions by DFT.
We further calculate for $\mathrm{ZnPc} / \mathrm{C}_{60}$ and $\mathrm{F}_{4} \mathrm{ZnPc} / \mathrm{C}_{60}$ the Coulomb binding energies of the energetically relaxed ion pairs and find that they differ up to several $10 \mathrm{meV}$ between $\mathrm{ZnPc}$ and $\mathrm{F}_{4} \mathrm{ZnPc}$ because of their different charge distributions ${ }^{43}$ (see Methods section and Supplementary Fig. 10a). We calculate from these parameters the expected variation of $E_{\mathrm{CT}}$ from $\mathrm{ZnPc}_{\mathrm{C}} \mathrm{C}_{60}$ to $\mathrm{F}_{4} \mathrm{ZnPc}: \mathrm{C}_{60}$ (dashed line in Fig. $4 \mathrm{~b}$ and Supplementary Fig. 10b). Experimental values of $E_{\mathrm{CT}}$ obtained from Gaussian fits to sensitively measured external quantum efficiency (see Supplementary Fig. 11) and electroluminescence spectra ${ }^{44}$. In good agreement with the calculations, the experimental values increase with $\mathrm{F}_{4} \mathrm{ZnPc}$ content (purple squares in Fig. $4 \mathrm{~b}$ ). However, the measured shift of $E_{\mathrm{CT}}$ is $0.1 \mathrm{eV}$ larger than expected from the variation of molecular parameters, which can be partly attributed to the larger static energetic disorder at high $\mathrm{ZnPc}$ contents, reducing $E_{\mathrm{CT}}$ (Supplementary Fig. 12). In addition to energetic disorder, charge-quadrupole interactions lower the effective IE of the donor and further reduce $E_{\mathrm{CT}}$ at large amounts of $\mathrm{ZnPc}$.

To verify the impact of charge-quadrupole interactions on $E_{\mathrm{CT}}$, we additionally fabricate solar cells with a planar heterojunction $(\mathrm{PHJ})$ between a $\mathrm{ZnPc}_{\mathrm{F}} \mathrm{F}_{4} \mathrm{ZnPc}$ blend layer with varying mixing ratio and a neat $\mathrm{C}_{60}$ layer. The $\mathrm{ZnPc}: \mathrm{F}_{4} \mathrm{ZnPc}$ layer is grown on p-doped BPAPF to ensure edge-on orientation of the donor molecules, causing a large change of IE induced by 

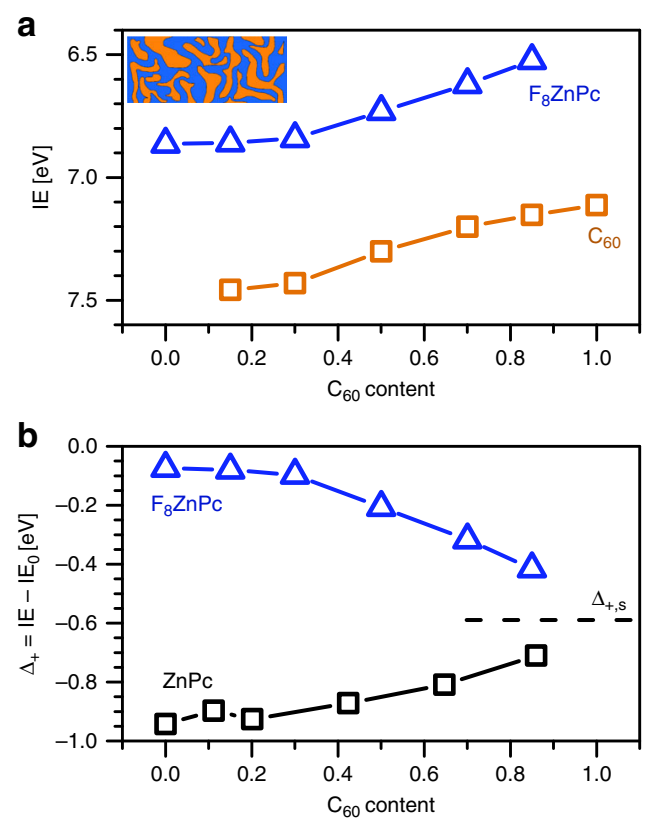

Fig. 3 Electronic levels in donor:acceptor blends: a IE of $\mathrm{F}_{8} \mathrm{ZnPc}$ and $\mathrm{C}_{60}$ at different molar contents of $C_{60}$, as obtained by UPS. $\mathbf{b}$ With increasing $C_{60}$ content, the electrostatic corrections $\Delta_{+}$of $\mathrm{ZnPc}$ and $\mathrm{F}_{8} \mathrm{ZnPc}$, being the difference in IE between the thin film and the gas-phase, shift towards $-0.6 \mathrm{eV}$. This value is expected for negligible charge-quadrupole interaction energy (see results in Fig. $2 \mathrm{~d}$ for very small film coverages). Experimental IE values of $\mathrm{ZnPc}: \mathrm{C}_{60}$ blends are taken from the study by Tietze et al. ${ }^{13}$

charge-quadrupole interactions (see Fig. 2b and Supplementary Fig. 9). At high $\mathrm{ZnPc}$ contents, the planar devices exhibit a significantly lower $E_{\mathrm{CT}}$ (green circles in Fig. $4 \mathrm{~b}$ ) than the ternary $\mathrm{BHJ}$ devices. This can be explained by extended long-range order, which increases the charge-quadrupole interaction energy and reduces IE of the donor for large $\mathrm{ZnPc}$ contents. The change of $E_{\text {Ст }}$ with donor mixing ratio directly influences $V_{\text {ос }}$ because charge carriers recombine via CT states at open-circuit ${ }^{9}$. Therefore, the different shifts of $E_{\mathrm{CT}}$ in $\mathrm{BHJ}$ and $\mathrm{PHJ}$ devices upon changing the donor content is reflected in a similar difference in $V_{\text {oc }}$ shift, demonstrating the relevance of charge-quadrupole interactions for important device parameters (Fig. 4c).

The generation efficiency of photocurrent depends on the dissociation barrier of CT excitons $\left(\Delta E_{\text {diss }}\right)$, which depends on the difference between $E_{\mathrm{CS}}$ and $E_{\mathrm{CT}}{ }^{11}$. We next discuss how quadrupole moments can induce electrostatic gradients at the donor-acceptor interface that lead to a lower $\Delta E_{\text {diss. }}$. In the case of a negative quadrupole component $Q_{\pi, \mathrm{D}}$ of the donor, charge -quadrupole interactions along the $\pi-\pi$-stacking direction reduce $\mathrm{IE}_{\mathrm{D}}$ and $E \mathrm{~A}_{\mathrm{D}}$ of donor molecules within the donor phase (green arrows in Fig. 5a). This shift is smaller at the interface with $\mathrm{C}_{60}$ due to the interaction with its neutral $Q_{\pi, \mathrm{A}}$ components. The negative $Q_{\pi, \mathrm{D}}$ influences the energies of acceptor molecules $\left(\mathrm{IE}_{\mathrm{A}}\right.$ and $E_{A}$ ) close to the interface, as indicated by the UPS measurements on donor-acceptor blends in Fig. 3. Therefore, the negative $Q_{\pi, \mathrm{D}}$ induces an electrostatic potential gradient from the donor to the acceptor phase, which directly reduces $\Delta E_{\text {diss. }}$.

To analyse $\Delta E_{\text {diss }}$ in $\mathrm{ZnPc:} \mathrm{F}_{4} \mathrm{ZnPc}: \mathrm{C}_{60}$ solar cells, we obtain the difference between $\mathrm{IE}_{\mathrm{D}}$ and $\mathrm{EA}_{\mathrm{A}}$ in ternary blends with UPS and take this as an estimate for $E_{C S}{ }^{45}$. The results in Fig. 5b show that $E_{\mathrm{CS}}$ increases more strongly with $Q_{\pi, \mathrm{D}}$ as compared to $E_{\mathrm{CT}}$, indicating a rise of $\Delta E_{\text {diss }}$ from high $\mathrm{ZnPc}$ to high $\mathrm{F}_{4} \mathrm{ZnPc}$ contents and verifying our considerations that a
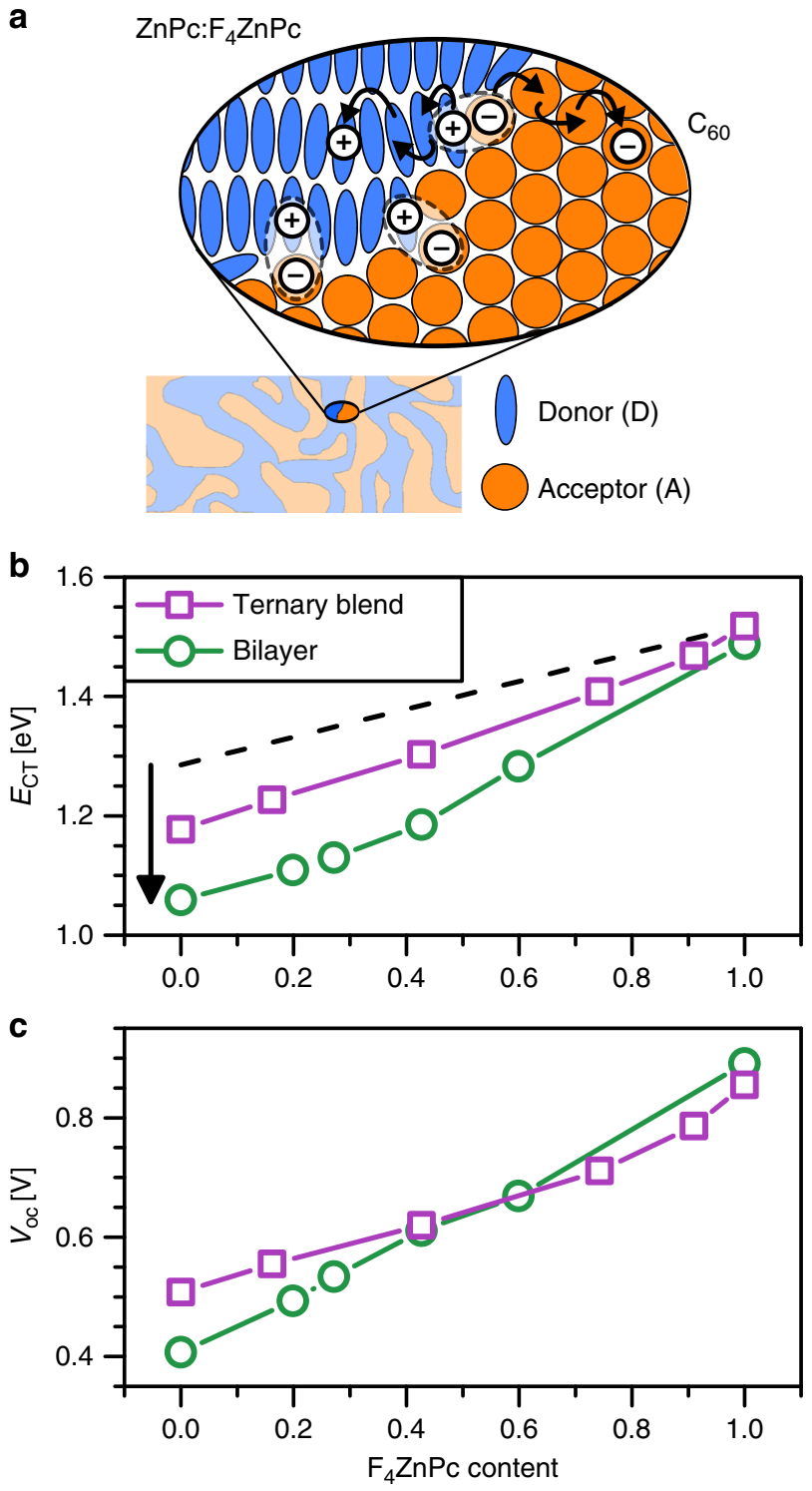

Fig. 4 Charge-transfer state energies at bulk and planar heterojunctions: a Simplified sketch of the interface between donor and acceptor phase in a bulk heterojunction (BHJ). The black arrows sketch a representative dissociation process of a charge-transfer (CT) exciton. $\mathbf{b}$ Experimental change of $E_{C T}$ as a function of the molar content of $\mathrm{F}_{4} \mathrm{ZnPc}$ in the intermixed donor phase ( $\mathrm{ZnPc}: \mathrm{F}_{4} \mathrm{ZnPc}$ ), forming a ternary $\mathrm{BHJ}$ (purple squares) or a planar heterojunction (green circles) with the acceptor $\mathrm{C}_{60}$. $E_{C T}$ values for the solar cells are obtained from Gaussian fits to sensitively measured external quantum efficiency (see Supplementary Fig. 11) and electroluminescence spectra 44 . The dashed black line is the shift of $E_{\mathrm{CT}}$, as calculated from the change of molecular parameters (see Eq. 4 in the Methods section). The black arrow highlights the decrease of $E_{\mathrm{CT}}$ at large $\mathrm{ZnPc}$ contents, which we attribute to charge-quadrupole interactions. c Change of $V_{\text {ocr }}$ measured at 1 sun illumination intensity, as a function of $\mathrm{F}_{4} \mathrm{ZnPc}$ content for both solar cell architectures. Note that the voltage losses $\left(\Delta V_{\mathrm{oc}}=E_{\mathrm{CT}} / e-V_{\mathrm{oc}}\right)$ are slightly lower for planar heterojunctions due to the reduced interfacial area ${ }^{58}$

charge-quadrupole interactions can reduce $\Delta E_{\text {diss. }}$ For PHJs, we observe a similar increase of $\Delta E_{\text {diss }}$ for higher $\mathrm{F}_{4} \mathrm{ZnPc}$ contents (Supplementary Fig. 13). We perform TDCF measurements ${ }^{11}$ on ternary solar cells to investigate how the increase of $\Delta E_{\text {diss }}$ affects the generation efficiency of free charge carriers. In this 
a
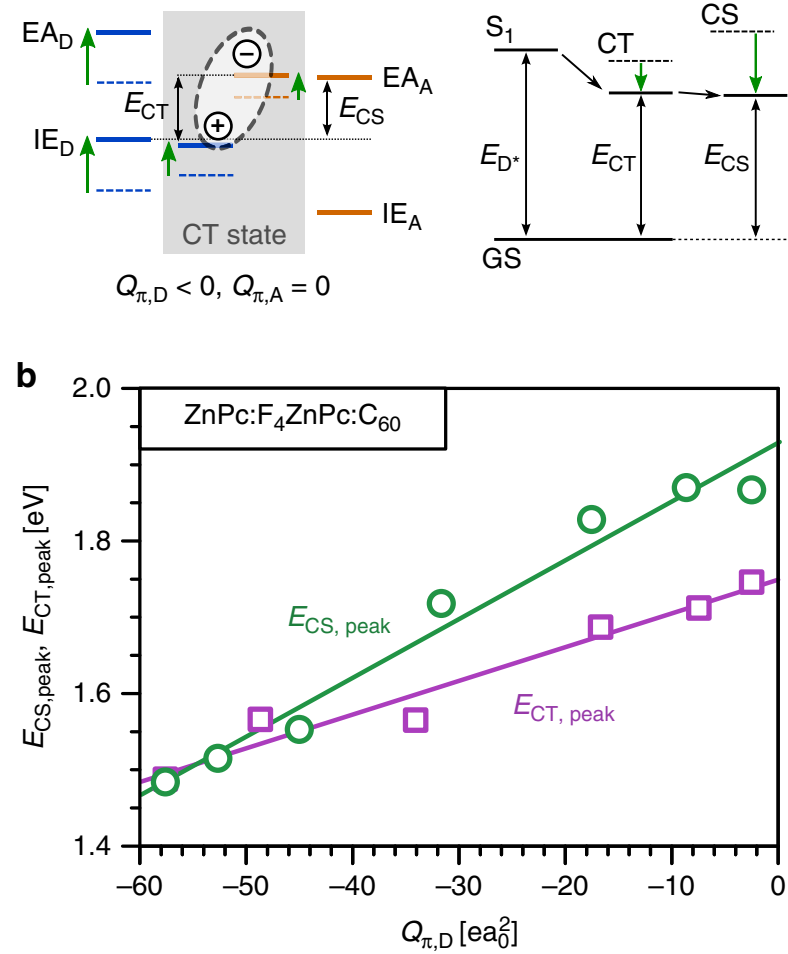
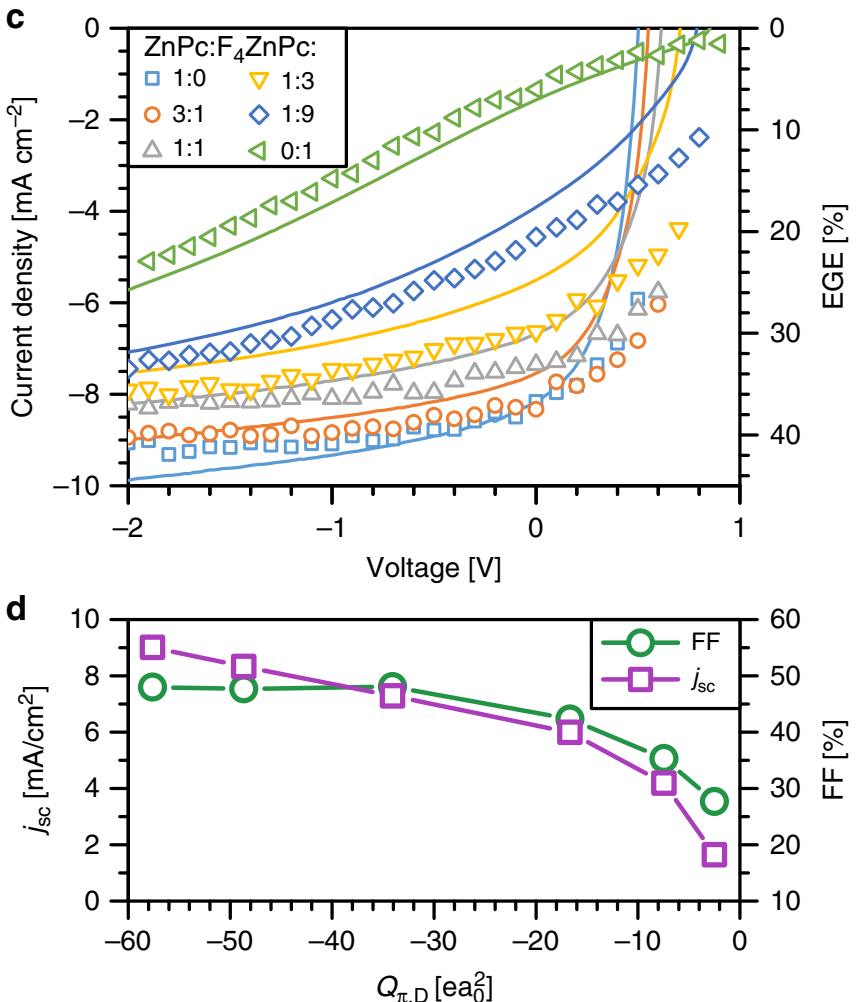

Fig. 5 CT exciton dissociation facilitated by quadrupole moments: a Simplified sketch of the donor and acceptor energy levels at the interface (CT state), highlighted by the grey area, and in the bulk of donor and acceptor phase for $Q_{\pi, D}=0$ (dashed lines) and $Q_{\pi, D}<0$ (solid lines). The green arrows illustrate the energy shifts due to charge-quadrupole interactions, reducing the CT dissociation barrier $\left(\Delta E_{\text {diss }}\right)$, i.e. the difference between the energy of separated charges $\left(E_{C S}\right)$ and $E_{C T}$. The right panel sketches the change of $E_{C T}$ and $E_{C S}$ together with the singlet state $\left(S_{1}\right)$ energy and the ground state $(G S)$. b $E_{C S, \text { peak }}$ estimated from UPS measurements, increases stronger with the averaged $Q_{\pi, D}$ as compared to the peak energy of the $C T$ state ( $E_{C T \text {,peak }}$ ), indicating an enhancement of $\Delta E_{\text {diss }}$ with increasing $Q_{\pi, D}$, i.e. with larger $F_{4} Z n P c$ contents. $E_{C S \text {,peak }}$ and $E_{C T \text {,peak }}$ are obtained from peak positions to exclude energetic disorder and experimental broadening from the analysis. Furthermore, $E_{\mathrm{CS} \text {, peak }}$ is reduced by $0.6 \mathrm{eV}$ to account for the polarisation difference between surface and bulk ${ }^{59,60}$. The solid lines are guides to the eye. c Time-delayed collection field (TDCF) measurements show that the reduction in photocurrent and fill factor (FF) for large $\mathrm{F}_{4} \mathrm{ZnPc}$ contents is caused by field-dependent photocurrent generation. The solid lines and left axis show the current-density/ voltage characteristics of the solar cells, whereas the symbols and right axis depict the external generation efficiency (EGE) of the photocurrent. $\mathbf{d}$ The increase of $\Delta E_{\text {diss }}$ with $Q_{\pi, D}$, as shown in $(\mathbf{b})$, causes a reduction of the short-circuit current density $\left(j_{s c}\right)$ and $\mathrm{FF}$ of the respective solar cells measured at 1 sun illumination conditions

method, donor molecules are excited by a short laser pulse. After a delay of $8 \mathrm{~ns}$, when all geminate recombination has taken place ${ }^{46,47}$ (Supplementary Fig. 14), the charges are extracted by applying a large negative bias voltage. As shown in Fig. 5c, the amount of collected charges follows the currentdensity/voltage characteristics of the solar cells, revealing that the reduced photocurrent at high $\mathrm{F}_{4} \mathrm{ZnPc}$ contents is predominantly due to a field-dependent free charge carrier generation ${ }^{11}$. We attribute the increased field-dependence to the increased $\Delta E_{\text {diss. }}$. The increase of $\Delta E_{\text {diss }}$ with $Q_{\pi, \mathrm{D}}$ causes a significant reduction of the device parameters $\mathrm{FF}$ and $j_{\mathrm{sc}}$ once $Q_{\pi, \mathrm{D}}$ is larger than $-30 e a_{0}^{2}$ (Fig. 5d). This finding explains the observation in previous studies where the use of $\mathrm{F}_{4}{ }^{\star} \mathrm{ZnPc}$ (a variant of $\mathrm{F}_{4} \mathrm{ZnPc}$, see Fig. 1 for the chemical structure) as donor in combination with $\mathrm{C}_{60}$ yields well-performing solar cells ${ }^{48}$. In contrast to $\mathrm{F}_{4} \mathrm{ZnPc}, \mathrm{F}_{4}{ }^{\star} \mathrm{ZnPc}$ has a more negative $Q_{\pi, \mathrm{D}}$ of $-41.6 e a_{0}^{2}$, being sufficiently low to ensure efficient CT exciton separation.

The correlation of FF and $j_{\mathrm{sc}}$ with $Q_{\pi}$ is not restricted to this particular system. We further calculate $Q_{\pi, D}$ and $Q_{\pi, \mathrm{A}}$ values for three donors (SubNc, $\mathrm{ZnPc}, \mathrm{F}_{4}{ }^{\star} \mathrm{ZnPc}$ ) each combined with one non-fullerene acceptor $\left(\mathrm{Cl}_{4} \mathrm{SubPc}\right.$ or $\left.\mathrm{Cl}_{6} \mathrm{SubPc}\right)$. In PHJ devices ${ }^{49}$, $\mathrm{FF}$ and $j_{\mathrm{sc}}$ increase for all donors when the acceptor $\mathrm{Cl}_{4} \mathrm{SubPc}$ is substituted by $\mathrm{Cl}_{6} \mathrm{SubPc}$, which can be explained by the increased difference between $Q_{\pi, \mathrm{D}}$ and $Q_{\pi, \mathrm{A}}$ (Supplementary Fig. 15). These results indicate that a precise adjustment of $Q_{\pi, A}$ being larger than $Q_{\pi, \mathrm{D}}$ should be considered when designing efficient non-fullerene acceptor molecules. For example, $Q_{\pi, \mathrm{A}}$ can be increased by adding electron withdrawing side groups to the acceptor. In literature, there are already examples of efficient acceptors having such side groups ${ }^{50,51}$, where a larger degree of fluorination resulted in an improved charge extraction ${ }^{52}$. Furthermore, the results of a recent study indicate that quadrupole moments also affect polymer solar cells. Here, an improved charge generation in P3HT/PCBM bilayer solar cells was observed when P3HT was oriented end-on ${ }^{42}$, which can be explained by the reduced relevance of the positive quadrupole component along the polymer chain (see quadrupole components of alpha-6T in Supplementary Table 1 for comparison). Therefore, quadrupole moments and molecular orientation should be taken more into account for the design of future photovoltaic materials such as small molecules, oligomers and polymers.

\section{Discussion}

In conclusion, we show that charge-quadrupole interactions along the $\pi$ - $\pi$-stacking direction can induce large electrostatic energy shifts to the electronic levels of molecular films, depending 
on molecular orientation and blend composition. Due to the sensitivity of the interaction energy to the local morphology, the electronic levels of blends strongly depend on the mixing ratio. Utilising the example of OSCs, we show the dependency of the charge-transfer state energy at donor-acceptor interfaces on the quadrupole component in $\pi-\pi$-stacking direction. Moreover, we present a strategy to tune the driving force for free-chargecarrier generation by adjusting the respective quadrupole components, which can be used to optimise non-fullerene acceptor molecules. A similar correlation is found for other materials than $\mathrm{ZnPc}$ derivatives, suggesting that our findings can be applied to a large variety of small molecules and polymers. These results highlight the necessity to consider the quadrupole moment as an important molecular parameter in future material design for high-performing organic semiconductor devices.

\section{Methods}

Ultraviolet photoelectron spectroscopy. The spectra are acquired by a PHOIBOS 100 analyser system (Specs, Berlin, Germany) at a base pressure of $10^{-11} \mathrm{mbar}$ using He I excitation lines $(21.22 \mathrm{eV})$ and an energy resolution of around $150 \mathrm{meV}$. By repeating the sample production under the same experimental conditions, the experimental error for the position of the obtained energetic states is estimated to be $50 \mathrm{meV}$. The Fermi level positions of all spectra are calibrated to the Fermi edges of the gold or silver substrates. All samples are thermally (co-)evaporated at rates of $0.1-0.2 \AA \mathrm{s}^{-1}$ in UHV at a base pressure of $10^{-8} \mathrm{mbar}$ using individual quartz crystal monitors for each material. As substrates, sputter-cleaned gold foils are used for face-on orientation and silver foils covered by $5 \mathrm{~nm}$ of an amorphous layer of $\mathrm{p}$ doped BPAPF (3wt\%, doped with NDP9) for edge-on orientation ${ }^{29}$. NDP9 is a commercial p-dopant supplied by Novaled GmbH, Germany. The layer thickness of the organic layers under investigation is always $20 \mathrm{~nm}$. IE values are obtained from the sum of the work function and the maximum position of the HOMO peak. The work function is extracted from the onset of the high binding energy cut-off.

X-ray scattering. The experiments are performed at the ESRF, France (beamline ID03), with a photon energy of $E=22.0 \mathrm{keV}$. The reciprocal space maps (RSM) are measured under grazing incidence geometry with an angle of incidence of $\alpha_{\mathrm{i}}=$ $0.07^{\circ}$. Each RSM is assembled from 16 single images recorded with a PILATUS $300 \mathrm{k}$ area detector. All measurements are performed in air. The samples are thermally (co-)evaporated at rates of $0.1-0.2 \AA \mathrm{s}^{-1}$ in UHV at a base pressure of $10^{-8} \mathrm{mbar}$ using individual quartz crystal monitors for each material. As substrates, glass substrates covered by $1 \mathrm{~nm}$ of chromium and $30 \mathrm{~nm}$ of gold are used for face-on orientation and glass substrates covered by $5 \mathrm{~nm}$ of an amorphous BPAPF are used for edge-on orientation ${ }^{29}$. The layer thickness of the $\mathrm{F}_{n} \mathrm{ZnPc}$ layers is $20 \mathrm{~nm}$.

Solar cell device preparation. The solar cells are thermally evaporated at ultrahigh vacuum (base pressure $<10^{-7} \mathrm{mbar}$ ) on a glass substrate with a pre-structured indium tin oxide (ITO) contact (Thin Film Devices, USA). The layer stacks of the ternary bulk heterojunction solar cells are: Glass/ITO/BPAPF:NDP9 $(40 \mathrm{~nm}, 5 \mathrm{wt}$ $\%) / \mathrm{ZnPc}: \mathrm{F}_{4} \mathrm{ZnPc}\left(5 \mathrm{~nm}\right.$, varying ratio) $/ \mathrm{ZnPc}: \mathrm{F}_{4} \mathrm{ZnPc:} \mathrm{C}_{60}(38 \mathrm{~nm}$, varying $\mathrm{ZnPc}$ : $\mathrm{F}_{4} \mathrm{ZnPc}$ ratio, $60 \mathrm{vol} \%$ of $\left.\mathrm{C}_{60}\right) / \mathrm{C}_{60}(15 \mathrm{~nm}) / \mathrm{C}_{60}: \mathrm{W}_{2}(\mathrm{hpp})_{4}(8 \mathrm{~nm}, 3 \mathrm{wt} \%) / \mathrm{Al}(100$ $\mathrm{nm})$. The layer stacks for planar heterojunctions are: Glass/ ITO/ BPAPF:NDP9 $(20 \mathrm{~nm}, 10 \mathrm{wt} \%) / \mathrm{ZnPc}: \mathrm{F}_{4} \mathrm{ZnPc}(10 \mathrm{~nm}$, varying ratio $) / \mathrm{C}_{60}(40 \mathrm{~nm}) / \mathrm{BPhen}(8 \mathrm{~nm}) /$ $\mathrm{Al}(100 \mathrm{~nm})$. NDP9 is a commercial p-dopant supplied by Novaled GmbH, Germany. All the organic materials were purified $2-3$ times by sublimation. The device area of $6.44 \mathrm{~mm}^{2}$ is defined by the geometrical overlap of the bottom and the top contact, verified with a profilometer. To avoid exposure to ambient conditions, the organic part of the device is covered by a small glass substrate which is glued on top. The relative content of donor and acceptor phases in ternary blends is estimated to have a precision of better than $\pm 5 \mathrm{wt} \%$.

Current-voltage characteristics. The current-voltage characteristics in dark and under solar illumination are measured with a source measure unit (Keithley 2400, USA) at room temperature. For the latter condition, the solar cells are illuminated with a spectrally mismatch-corrected intensity of $100 \mathrm{~mW} \mathrm{~cm}^{-2}$ (AM1.5G) provided by a sun simulator (16 S-150 V.3 Solar Light Co., USA) and masked to avoid edge effects and to precisely define the area. The intensity is monitored with a calibrated Hamamatsu S1337 silicon photodiode.

Sensitive external quantum efficiency (sEQE). The light of a quartz halogen lamp $(50 \mathrm{~W})$ is chopped at $141 \mathrm{~Hz}$ and coupled into a monochromator (Newport Cornerstone $2601 / 4 \mathrm{~m}$, USA). The resulting monochromatic light is focused onto the solar cell, of which the short-circuit current is fed to a current pre-amplifier before it is analysed with a lock-in amplifier (Signal Recovery 7280 DSP, USA). The time constant of the lock-in amplifier was chosen to be $0.5 \mathrm{~s}$ or $1.0 \mathrm{~s}$ and the amplification of the pre-amplifier was increased to resolve low photocurrents.
The EQE is determined by dividing the photocurrent of the OSC by the flux of incoming photons, which was measured using a calibrated $\mathrm{Si}$ and InGaAs photodiode. The measurements are performed at room temperature.

Electroluminescence (EL). EL measurements were obtained at room temperature with an Andor SR393i-B spectrometer equipped with a cooled Si and cooled InGaAs CCD detector array (DU420A-BR-DD and DU491A-1.7, UK). The spectral response of the setup was calibrated with a reference lamp (Oriel 63355). The emission spectrum of the OSCs was recorded at different injection currents with respect to voltages, which were lower than or at least similar to the $V_{\mathrm{oc}}$ of the device at 1 sun illumination.

Time-delayed collection field. In TDCF measurements, the device is excited while held at a varying pre-bias and after a delay time of $8 \mathrm{~ns}$ a collection bias of $2.5 \mathrm{~V}$ is applied. The excitation was generated with a diode-pumped, Q-switched Nd:YAG laser (NT242, EKSPLA, $500 \mathrm{~Hz}$ rep-rate, $5.2 \mathrm{~ns}$ pulse duration, $590 \mathrm{~nm}$ wavelength). Also, to compensate for the internal latency of the pulse generator, the laser pulse was delayed and homogeneously scattered in an $85 \mathrm{~m}$ long silica fibre (LEONI). An Agilent 81150A pulse generator was employed to apply the pre-bias and collection bias to a homebuilt amplifier directly connected to the sample. The current through the device was measured via a grounded $10 \Omega$ resistor in series with the sample and recorded with an Agilent DSO9104H oscilloscope.

Density functional theory. The simulations are performed to calculate the molecular quadrupole moments, the ionisation energy, and the intramolecular relaxation energy in gas-phase of the relevant molecules. The quadrupole tensor components $Q_{i j}$ are obtained from following definition:

$$
Q_{i j}=\int p(\mathbf{r}) \cdot\left(3 r_{i} r_{j}-|\mathbf{r}|^{2} \delta_{i j}\right) \cdot \mathbf{d}^{3} \mathbf{r} .
$$

The ionisation energy was determined as the difference of the total energy of the positively charged molecule and the neutral molecule in the relaxed geometry of the neutral molecule. The intramolecular relaxation energy is determined as the difference in total energy between the negatively (positively) charged molecule in its optimised geometry and its energy in the geometry of the neutral molecule. We used the M06-2x exchange-correlation functional ${ }^{53}$ and the correlation-consistent basis set cc-pVTZ ${ }^{54}$ as implemented in the computational chemistry package NWChem ${ }^{55}$.

Charge-quadrupole interaction energy calculation. The energy is calculated for a given molecule at site $\mathbf{r}_{j}$ as a discrete sum including all other molecules (at sites $\mathbf{r}_{i}$ ) in the considered geometry, according to

$$
E_{\mathrm{Q}}\left(\mathbf{r}_{j}\right)=\sum_{i, k} \frac{q_{j, k}}{8 \pi \epsilon_{0} \epsilon_{\mathrm{r}}} \frac{\left(\mathbf{r}_{i}-\mathbf{r}_{j}-\boldsymbol{\tau}_{k}\right) \cdot \mathbf{Q}_{i} \cdot\left(\mathbf{r}_{i}-\mathbf{r}_{j}-\boldsymbol{\tau}_{k}\right)}{\left|\mathbf{r}_{i}-\mathbf{r}_{j}-\boldsymbol{\tau}_{k}\right|^{5}}
$$

with the quadrupole tensor $\mathbf{Q}_{i}$ of molecule $i$ and the relative dielectric permittivity (assuming $\varepsilon_{\mathrm{r}}=2.8^{56}$ for all $\mathrm{F}_{n} \mathrm{ZnPcs}$ ). Hereby, $q_{j, k}$ is the fractional excess charge at atom $k$ of the molecule $j$ and $\mathbf{r}_{j}+\boldsymbol{\tau}_{k}$ is its position. The quadrupole tensor and the fractional excess charges are obtained in gas phase for all molecules in their respective relaxed structures. The charge distributions and the resulting quadrupole moments might slightly differ in the film phase due to the surrounding polarisable medium. The film structure was generated according to the crystal structure of $\mathrm{CuPC}^{57}$ (see Supplementary Figs. 1-4). We assume a simplified orthorhombic lattice and take the intermolecular distances (approximately constan $3.8 \AA$ and $13.5 \AA$ ) from literature ${ }^{28,30}$. For face-on geometry, a film thickness of 20 $\mathrm{nm}$ implies that we take into account 53 layers along the surface normal. We restrict the summation in lateral directions to a large area of $400 \mathrm{~nm} \times 400 \mathrm{~nm}$, which is sufficient for convergence. For edge-on geometry, we have 15 layers in the direction of the surface normal for a $20 \mathrm{~nm}$ film, while the lateral dimension of the integration region is equally big. To investigate the relevant range for the interaction energy, we vary the summation in lateral direction between 10 and $200 \mathrm{~nm}$ (Supplementary Fig. 5). In addition, we reduced the thickness of the film in edge-on orientation from 20 to $3 \mathrm{~nm}$ and observe an increase of the interaction energy by $10 \%$ for $\mathrm{ZnPc}$, in good agreement to the change of IE observed in experiment (Fig. 2d)

Energy change of CT states due to molecular parameters. We estimate the variation of the CT state energy $\left(E_{\mathrm{CT}}\right)$ from the change of molecular parameters when replacing $\mathrm{ZnPc}$ with $\mathrm{F}_{4} \mathrm{ZnPc}$ using following expression:

$$
E_{\mathrm{CT}}=\mathrm{IE}_{0, \mathrm{D}}-\mathrm{EA}_{0, \mathrm{~A}}-E_{\text {coul }, \mathrm{CT}}-\lambda_{\mathrm{D}}-\lambda_{\mathrm{A}},
$$

where $\mathrm{IE}_{0, \mathrm{D}}$ is the gas-phase ionisation energy of the donor $\left(\mathrm{ZnPc} / \mathrm{F}_{4} \mathrm{ZnPc}\right)$ and $\mathrm{EA}_{0, \mathrm{~A}}$ is the gas-phase electron affinity of the acceptor $\left(\mathrm{C}_{60}\right) . E_{\text {coul,CT }}$ is the Coulomb binding energy between the donor cation and the acceptor anion, which is screened by a mean dielectric constant of $\varepsilon_{\mathrm{r}}=3.6$, obtained from $\varepsilon_{\mathrm{r}}=2.8$ for $\mathrm{ZnPc}$ and $\varepsilon_{\mathrm{r}}=2.8$ for $\mathrm{C}_{60}{ }^{56}$. For a detailed description how $E_{\text {coul,CT }}$ is obtained, we refer the reader to a our previous publication ${ }^{43} . \lambda_{\mathrm{D}}$ is the intramolecular relaxation energy of the donor cation and $\lambda_{\mathrm{A}}$ is the intramolecular relaxation energy of the 
acceptor anion. All values are obtained from DFT simulations. To compare with the experimental data in Fig. $4 \mathrm{~b}$, we additionally subtract a polarisation energy of $2.1 \mathrm{eV}$.

\section{Data availability}

All the data supporting the findings of this study are available within the article, its Supplementary Information files, or from the corresponding authors upon reasonable request.

\section{Code availability}

The code used for the calculation of the charge-quadrupole interaction energy is available from the corresponding authors upon reasonable request.

Received: 8 April 2019 Accepted: 11 May 2019

Published online: 05 June 2019

\section{References}

1. Forrest, S. R. The path to ubiquitous and low-cost organic electronic appliances on plastic. Nature 428, 911-918 (2004).

2. Someya, T. et al. A large-area, flexible pressure sensor matrix with organic field-effect transistors for artificial skin applications. Proc. Natl Acad. Sci. USA 101, 9966-9970 (2004)

3. Mannsfeld, S. C. B. et al. Highly sensitive flexible pressure sensors with microstructured rubber dielectric layers. Nat. Mater. 9, 859-864 (2010)

4. Søndergaard, R. R., Hösel, M. \& Krebs, F. C. Roll-to-roll fabrication of large area functional organic materials. J. Polym. Sci. Part B Polym. Phys. 51, 16-34 (2013).

5. Zahn, D. R. T., Gavrila, G. N. \& Gorgoi, M. The transport gap of organic semiconductors studied using the combination of direct and inverse photoemission. Chem. Phys. 325, 99-112 (2006).

6. Krause, S., Casu, M. B., Schöll, A. \& Umbach, E. Determination of transport levels of organic semiconductors by UPS and IPS. New J. Phys. 10, 085001 (2008).

7. Vandewal, K. et al. Efficient charge generation by relaxed charge-transfer states at organic interfaces. Nat. Mater. 13, 63-68 (2014).

8. Sini, G. et al. On the molecular origin of charge separation at the donor -acceptor interface. Adv. Energy Mater. 8, 1702232 (2018).

9. Benduhn, J. et al. Impact of triplet excited states on the open-circuit voltage of organic solar cells. Adv. Energy Mater. 8, 1800451 (2018).

10. Vandewal, K., Tvingstedt, K., Gadisa, A., Inganäs, O. \& Manca, J. V. On the origin of the open-circuit voltage of polymer-fullerene solar cells. Nat. Mater. 8, 904-909 (2009).

11. Albrecht, S. et al. On the efficiency of charge transfer state splitting in polymer:fullerene solar cells. Adv. Mater. 26, 2533-2539 (2014)

12. Bartesaghi, D. et al. Competition between recombination and extraction of free charges determines the fill factor of organic solar cells. Nat. Commun. 6, 7083 (2015).

13. Tietze, M. L. et al. Correlation of open-circuit voltage and energy levels in zinc-phthalocyanine: C60 bulk heterojunction solar cells with varied mixing ratio. Phys. Rev. B 88, 085119 (2013).

14. Duhm, S. et al. Orientation-dependent ionization energies and interface dipoles in ordered molecular assemblies. Nat. Mater. 7, 326-332 (2008).

15. Graham, K. R. et al. The roles of structural order and intermolecular interactions in determining ionization energies and charge-transfer state energies in organic semiconductors. Adv. Energy Mater. 6, 1601211 (2016).

16. Poelking, C. et al. Impact of mesoscale order on open-circuit voltage in organic solar cells. Nat. Mater. 14, 434-439 (2015).

17. Poelking, C. \& Andrienko, D. Long-range embedding of molecular ions and excitations in a polarizable molecular environment. J. Chem. Theory Comput. 12, 4516-4523 (2016).

18. D'Avino, G. et al. Electrostatic phenomena in organic semiconductors: fundamentals and implications for photovoltaics. J. Phys. Condens. Matter 28, 433002 (2016).

19. Yoshida, H., Yamada, K., Tsutsumi, J. \& Sato, N. Complete description of ionization energy and electron affinity in organic solids: determining contributions from electronic polarization, energy band dispersion, and molecular orientation. Phys. Rev. B 92, 075145 (2015).

20. Schwarze, $M$. et al. Band structure engineering in organic semiconductors. Science 352, 1446-1449 (2016).

21. Ueno, N. Tuning organic band structures with Coulomb interactions. Science 352, 1395-1396 (2016).

22. Poelking, C. \& Andrienko, D. Design rules for organic donor-acceptor heterojunctions: pathway for charge splitting and detrapping. J. Am. Chem. Soc. 137, 6320-6326 (2015).
23. Verlaak, S. et al. Electronic structure and geminate pair energetics at organic - organic interfaces: the case of Pentacene/C60 heterojunctions. Adv. Funct. Mater. 19, 3809-3814 (2009).

24. Sato, N., Seki, K. \& Inokuchi, H. Polarization energies of organic solids determined by ultraviolet photoelectron spectroscopy. J. Chem. Soc. Faraday Trans. 2, 1621 (1981).

25. Schlettwein, D. et al. Electronic energy levels in individual molecules, thin films, and organic heterojunctions of substituted phthalocyanines. J. Phys. Chem. B 105, 4791-4800 (2001).

26. Tsiper, E. V. \& Soos, Z. G. Charge redistribution and polarization energy of organic molecular crystals. Phys. Rev. B 64, 195124 (2001).

27. Jackson, J. D. Classical Electrodynamics (Wiley, New York, 1998).

28. Schünemann, C. et al. Zinc phthalocyanine-influence of substrate temperature, film thickness, and kind of substrate on the morphology. Thin Solid Films 519, 3939-3945 (2011)

29. Schünemann, C. et al. Evaluation and control of the orientation of small molecules for strongly absorbing organic thin films. J. Phys. Chem. C 117, 11600-11609 (2013)

30. Brendel, M. et al. The effect of gradual fluorination on the properties of FnZnPc thin films and FnZnPc/C60 bilayer photovoltaic cells. Adv. Funct. Mater. 25, 1565-1573 (2015)

31. Yamada, K. et al. Impact of the molecular quadrupole moment on ionization energy and electron affinity of organic thin films: experimental determination of electrostatic potential and electronic polarization energies. Phys. Rev. B 97, 245206 (2018).

32. Banerjee, R. et al. Evidence for knetically limited thickness dependent phase separation in organic thin film blends. Phys. Rev. Lett. 110, 185506 (2013).

33. Moench, T. et al. Influence of meso and nanoscale structure on the properties of highly efficient small molecule solar cells. Adv. Energy Mater. 6, 1501280 (2016).

34. Mönch, T. et al. Experimental and theoretical study of phase separation in ZnPc:C60 blends. Org. Electron. 27, 183-191 (2015).

35. Schünemann, C. et al. Phase separation analysis of bulk heterojunctions in small-molecule organic solar cells using zinc-phthalocyanine and C60. Phys. Rev. B 85, 245314 (2012).

36. Lorch, C. et al. Controlling length-scales of the phase separation to optimize organic semiconductor blends. Appl. Phys. Lett. 107, 201903 (2015).

37. Abtahi, A. et al. Effect of halogenation on the energetics of pure and mixed phases in model organic semiconductors composed of anthradithiophene derivatives and C60. J. Phys. Chem. C 122, 4757-4767 (2018).

38. Chen, W. et al. Molecular orientation dependent energy level alignment at organic-organic heterojunction interfaces. J. Phys. Chem. C 113, 12832-12839 (2009)

39. Zhong, J. Q. et al. Effect of gap states on the orientation-dependent energy level alignment at the DIP/F16CuPc donor-acceptor heterojunction interfaces. J. Phys. Chem. C 115, 23922-23928 (2011)

40. Bussolotti, F., Kera, S., Kudo, K., Kahn, A. \& Ueno, N. Gap states in pentacene thin film induced by inert gas exposure. Phys. Rev. Lett. 110, 267602 (2013).

41. Han, W., Yoshida, H., Ueno, N. \& Kera, S. Electron affinity of pentacene thin film studied by radiation-damage free inverse photoemission spectroscopy. Appl. Phys. Lett. 103, 123303 (2013).

42. Wang, F. et al. Effects of end-on oriented polymer chains at the donor/ acceptor interface in organic solar cells. J. Mater. Chem. A 6, 22889-22898 (2018).

43. Schwarze, M. et al. Molecular parameters responsible for thermally activated transport in doped organic semiconductors. Nat. Mater. 18, 242-248 (2019).

44. Vandewal, K., Tvingstedt, K., Gadisa, A., Inganäs, O. \& Manca, J. V. Relating the open-circuit voltage to interface molecular properties of donor:acceptor bulk heterojunction solar cells. Phys. Rev. B 81, 125204 (2010).

45. Widmer, J., Tietze, M., Leo, K. \& Riede, M. Open-circuit voltage and effective gap of organic solar cells. Adv. Funct. Mater. 23, 5814-5821 (2013).

46. Armin, A., Durrant, J. R. \& Shoaee, S. Interplay between triplet-, singletcharge transfer states and free charge carriers defining bimolecular recombination rate constant of organic solar cells. J. Phys. Chem. C 121, 13969-13976 (2017).

47. Collado-Fregoso, E. et al. Energy-gap law for photocurrent generation in fullerene-based organic solar cells: the case of low-donor-content blends. J. Am. Chem. Soc. 141, 2329-2341 (2019).

48. Meiss, J. et al. Fluorinated zinc phthalocyanine as donor for efficient vacuumdeposited organic solar cells. Adv. Funct. Mater. 22, 405-414 (2012).

49. Cnops, K. et al. Energy level tuning of non-fullerene acceptors in organic solar cells. J. Am. Chem. Soc. 137, 8991-8997 (2015).

50. Hou, J., Inganäs, O., Friend, R. H. \& Gao, F. Organic solar cells based on nonfullerene acceptors. Nat. Mater. 17, 119-128 (2018). 
51. Che, X., Li, Y., Qu, Y. \& Forrest, S. R. High fabrication yield organic tandem photovoltaics combining vacuum- and solution-processed subcells with $15 \%$ efficiency. Nat. Energy 3, 422-427 (2018).

52. Fan, B. et al. Fine-tuning of the chemical structure of photoactive materials for highly efficient organic photovoltaics. Nat. Energy 3, 1051-1058 (2018).

53. Zhao, Y. \& Truhlar, D. G. The M06 suite of density functionals for main group thermochemistry, thermochemical kinetics, noncovalent interactions, excited states, and transition elements: two new functionals and systematic testing of four M06-class functionals and 12 other function. Theor. Chem. Acc. 120 215-241 (2008).

54. Dunning, T. H. Jr. Gaussian basis sets for use in correlated molecular calculations. I. The atoms boron through neon and hydrogen. J. Chem. Phys. 90, 1007 (1989).

55. Valiev, M. et al. NWChem: a comprehensive and scalable open-source solution for large scale molecular simulations. Comput. Phys. Commun. 181, 1477-1489 (2010).

56. Scholz, R. et al. Quantifying charge transfer energies at donor-acceptor interfaces in small-molecule solar cells with constrained DFTB and spectroscopic methods. J. Phys. Condens. Matter 25, 473201 (2013)

57. Hoshino, A., Takenaka, Y. \& Miyaji, H. Redetermination of the crystal structure of a-copper phthalocyanine grown on KCl. Acta Crystallogr. B59, 393-403 (2003).

58. Vandewal, K. et al. Increased open-circuit voltage of organic solar cells by reduced donor-acceptor interface area. Adv. Mater. 26, 3839-3843 (2014).

59. Yoshida, H. \& Sato, N. A precise analysis of the core-level energy difference between the surface and bulk region of organic semiconductor thin films. J. Phys. Chem. C 116, 10033-10038 (2012).

60. Gaul, C. et al. Insight into doping efficiency of organic semiconductors from the analysis of the density of states in n-doped C60 and ZnPc. Nat. Mater. 17, 439-444 (2018).

\section{Acknowledgements}

We thank Beatrice Beyer for providing $\mathrm{F}_{4} \mathrm{ZnPc}$ and $\mathrm{F}_{16} \mathrm{ZnPc}$. M.S. acknowledges financial support by the German Research Foundation (DFG) through the project MatWorldNet LE-747/44-1. F.O. would like to thank the DFG for financial support (project OR-349/1). The TDCF setup in Potsdam was partially financed by the DFG through INST 336/94-1 FUGG. This work was supported by the German Federal Ministry for Education and Research (BMBF) grant InterPhase (FKZ 13N13661) and through the InnoProfile project "Organische p-i-n Bauelemente 2.2" (03IPT602X). Moreover, the project received funding from the NMP-20-2014- "Widening materials models" programme under Grant Agreement No. 646259 (MOSTOPHOS). K.L. is a fellow of the Canadian Institute for Advanced Research (CIFAR). We gratefully acknowledge grants for computing time from the Zentrum für Informationsdienste und Hochleistungsrechnen Dresden (ZIH). The X-ray scattering experiments were performed on beamline ID3 at the European Synchrotron Radiation Facility (ESRF), Grenoble, France. We are grateful to Maciej
Jankowski for providing assistance at beamline ID3. We acknowledge support by the Open Access Publication Funds of the SLUB/TU Dresden.

\section{Author contributions}

M.S. designed the study and acquired most of the UPS data. K.S.S., C.G. and R.S. performed DFT simulations. K.O. acquired further UPS data. F.O. calculated charge-quadrupole interaction energies. A.H. performed X-ray scattering experiments. J.B., M.S. and K.V. designed the solar cell devices and J.B. did the characterisation. J.B. and J.K. performed sensitive EQE measurements, whereas J.B. evaluated the data. L.P.T. performed TDCF and EL measurements on ternary BHJ solar cells. S.R. performed EL measurements on PHJ solar cells. M.L. synthesised $F_{8}$ ZnPc. K.L., F.O., K.V., G.C., F.S. and D.N. supervised different parts of the study. D.A. and C.P. valuably contributed to the physical understanding of the underlying electrostatic interactions. M.S., F.O. and K.L. wrote the manuscript and all authors contributed to discussions and finalising the manuscript.

\section{Additional information}

Supplementary Information accompanies this paper at https://doi.org/10.1038/s41467019-10435-2.

Competing interests: The authors declare no competing interests.

Reprints and permission information is available online at http://npg.nature.com/ reprintsandpermissions/

Journal peer review information: Nature Communications thanks the anonymous reviewer(s) for their contribution to the peer review of this work. Peer reviewer reports are available.

Publisher's note: Springer Nature remains neutral with regard to jurisdictional claims in published maps and institutional affiliations.

(c) (i) Open Access This article is licensed under a Creative Commons Attribution 4.0 International License, which permits use, sharing adaptation, distribution and reproduction in any medium or format, as long as you give appropriate credit to the original author(s) and the source, provide a link to the Creative Commons license, and indicate if changes were made. The images or other third party material in this article are included in the article's Creative Commons license, unles indicated otherwise in a credit line to the material. If material is not included in the article's Creative Commons license and your intended use is not permitted by statutory regulation or exceeds the permitted use, you will need to obtain permission directly from the copyright holder. To view a copy of this license, visit http://creativecommons.org/ licenses/by/4.0\%

(c) The Author(s) 2019 